\title{
Hydrodehalogenation of Halogenated Aryl Ketones under Multiphase Conditions. 6. pH Effect on the Chemoselectivity and Preliminary Mechanistic Investigation
}

\author{
Alvise Perosa, Maurizio Selva, and Pietro Tundo*,† \\ Dipartimento di Scienze Ambientali, Università Ca' Foscari, Dorsoduro 2137, 30123 Venezia, I taly
}

Received November 30, 1998

\begin{abstract}
A model halogenated aryl ketone (4-chloropropiophenone 1 ) was subjected to mild catalytic hydrodehalogenation $\left(\mathrm{p}\left[\mathrm{H}_{2}\right]=1 \mathrm{~atm}, \mathrm{~T}=50^{\circ} \mathrm{C}\right)$ in a multiphase system consisting of isooctane and an alkaline aqueous phase, in the presence of onium salts as phase-transfer agents. The chemosel ectivity of the reaction was sharply influenced by the $\mathrm{KOH}$ concentration of the aqueous phase: thus, the formation of 1-phenyl-1-propanol $\mathbf{4}$ was favored in a highly alkaline pH range (> 13.5 by $50 \%$ to $5 \% \mathrm{KOH}_{\text {aq }}$ ). Instead, up to $95 \%$ selectivity toward phenyl ethyl ketone $\mathbf{2}$ was observed if the $\mathrm{pH}$ was controlled $(8<\mathrm{pH}<13)$ by adjusting the $\mathrm{KOH}_{\mathrm{aq}}$ concentration in the range between 5 and $1 \%$. Further investigation of the $\mathrm{pH}$ effect indicated that an acidic aqueous phase directed the reaction chemoselectivity toward the formation of products arising from aromatic ring reduction (i.e., cyclohexyl ethyl ketone $\mathbf{7}$ and 1-cyclohexyl-1-propanol 8) rather than from carbonyl hydrogenation or hydrogenolysis. Also aliphatic amines, used in place of the PT onium salt, afforded good selectivities toward 2, although not as high as the ones obtained with Aliquat 336.
\end{abstract}

\section{Introduction}

We previously reported that by operating in a multiphase system composed of an alkal ine aqueous phase and a hydrocarbon solvent such as isooctane, and in the presence of onium salts and of a phase-transfer (PT) agent, hal ogenated aromatics undergo facile and sel ective Pd-catalyzed hydrodehalogenation to afford the corresponding dehalogenated aromatics, under mild conditions $\left(\mathrm{p}\left[\mathrm{H}_{2}\right]=1 \mathrm{~atm}, \mathrm{~T}=50^{\circ} \mathrm{C}\right) .{ }^{1-3}$ Even sterically hindered aryl halides could be rapidly reduced to the corresponding aromatic hydrocarbons. ${ }^{2}$ When polyfunctionalized substrates such as halogenated aryl ketones were subjected to those conditions, we observed that the presence of the PT agent induced very high chemoselectivity: hydrodehalogenation took place without any further reduction of the carbonyl or of the aromatic moiety. ${ }^{4}$ For example, the reaction of $\mathrm{p}$-chloropropiophenone carried out over $\mathrm{Pd} / \mathrm{C}$ in the presence of Aliquat 336 (tricaprylmethylammonium chloride, A336) afforded selectively propiophenone when the onium salt was used.

More recently we reported that also by using $\mathrm{Pt} / \mathrm{C}$ as a catalyst, the chemoselectivity of the multiphase hydrodehalogenation of haloaromatics was sharply modified by the presence of a PT agent. ${ }^{5}$ Thus, while using A336, the hydrodehalogenation of haloaromatic ketones could yield selectively the corresponding benzylic alcohols (without reduction of the hydroxyl or aromatic moiety),

† Phone: +39041 257 8642. Fax: +39041 257 8620. e-mail: tundop@unive.it.

(1) Marques, C. A.; Selva, M.; Tundo, P. J . Org. Chem. 1993, 58, 5256.

(2) Marques, C. A.; Selva, M.; Tundo, P. J . Org. Chem. 1994, 59, 3430.

(3) Marques, C. A.; Selva, M.; Tundo, P. J . Mol. Catal. A: Chemical 1995, 96, 301.

(4) Marques, C. A.; Selva, M.; Tundo, P. J . Org. Chem. 1995, 60, 2430.

(5) Selva, M.; Tundo, P.; Perosa, A. J . Org. Chem. 1998, 63, 3266. and a wide range of reduction products was observed in the absence of the onium salt. Moreover, we also showed that an accurate control of the ratio PT agent/platinum was necessary in order to achieve the optimal compromise between selectivity and reaction time. A similar behavior, although with slower rates, was observed also for other hydrophilic PT agents such as triethylbenzylammonium chloride (TEBA) and a polyethyleneglycol such as $\mathrm{CH}_{3}\left(\mathrm{CH}_{2}\right)_{11} \mathrm{O}\left(\mathrm{CH}_{2} \mathrm{CH}_{2} \mathrm{O}\right)_{23} \mathrm{H}$ (Brij 35). Accordingly, both the chemical structure and the properties of the PT agent were suggested to play a role in determining the outcome of the hydrodehalogenation reaction.

Herein we describe further results in the study of the hydrodehalogenation reaction: particularly, concerning the effect on the chemoselectivity observed by varying the $\mathrm{pH}$ of the aqueous phase. Attempts to rationalize our observations by proposing a possible mode of action of the PT agent will also be discussed.

\section{Results and Discussion}

1. Multiphase System. To study the effect of the $\mathrm{KOH}_{\mathrm{aq}}$ concentration on the multiphase hydrodehalogenation reaction, a series of reactions was conducted by choosing 4-chloropropiophenone $\mathbf{1}$ as a model substrate. Experiments were run at $50^{\circ} \mathrm{C}$ : a mixture of $7 \mathrm{~mL}$ of an isooctane solution of the substrate $(0.07 \mathrm{M}), \mathrm{a} \mathrm{KOH}_{\mathrm{aq}}$ solution ( $4 \mathrm{~mL}$ ) of varying concentrations (from 5 to $0.5 \%$ in weight), the $5 \% \mathrm{Pt} / \mathrm{C}$ catalyst (Cl/Pt ratio of 32.6), and the PT agent (see Tables 1 and 2 for the amounts) was magnetically stirred while $\mathrm{H}_{2}$ was bubbled at atmospheric pressure (approximately $1 \mathrm{~mL} / \mathrm{min}$ ) into the reaction vessel. The choice of the reaction conditions and of the PT agents was dictated by the previous results that gave the best selectivity toward phenyl propanol $4^{5}$

The results are reported in Table 1, where the reaction times refer to when maximum selectivity toward $\mathbf{4}$ and 
Table 1. Hydrodehalogenation of 4-Chloropropiophenone 1 under Multiphase Conditions (Isooctane/ $\mathbf{K O H}_{\mathrm{aq}}$ ), Using $\mathbf{5 \%} \mathrm{Pt} / \mathrm{C}^{\mathrm{a}}$

\begin{tabular}{|c|c|c|c|c|c|c|c|c|c|}
\hline \multirow[b]{2}{*}{ run } & \multirow[b]{2}{*}{ aq phase } & \multirow[b]{2}{*}{ PT agent } & \multirow{2}{*}{$\begin{array}{l}\text { time } \\
\text { (min) }\end{array}$} & \multicolumn{6}{|c|}{ products (\% by GC) } \\
\hline & & & & 2 & 3 & 4 & 5 & 6 & 7 \\
\hline $1^{5}$ & $\mathrm{KOH}$ & A336 $53 \mathrm{mg}$ & 60 & & 6 & 94 & & & \\
\hline $2^{5}$ & $\mathrm{KOI}$ & TEE & 720 & & 5 & 95 & & & \\
\hline 3 & $\mathrm{KOH}$ & A336 $72 \mathrm{mg}$ & 360 & 12 & & 85 & & & \\
\hline 4 & $\mathrm{KOH}_{3}$ & TEBA $18 \mathrm{mg}$ & 360 & 15 & & 80 & & & 2 \\
\hline 5 & $\mathrm{KOH}$ & $72 \mathrm{mg}$ & 17 & 95 & & 3 & & & 2 \\
\hline 6 & $\mathrm{KOH}$ & $18 \mathrm{mg}$ & 20 & 89 & & 10 & & & 1 \\
\hline 7 & $\mathrm{KOH}_{\mathrm{a}}$ & none & 17 & 51 & & 31 & 6 & 11 & 1 \\
\hline
\end{tabular}

a Conditions: $\mathrm{T}=50{ }^{\circ} \mathrm{C} ; \mathrm{Cl} / \mathrm{Pt}=32.6 ; \mathrm{Pt} / \mathrm{C}$ (5\%) $0.0586 \mathrm{~g} ; 7$ $\mathrm{mL}$ of $0.07 \mathrm{M} 1$ in isooctane; $4 \mathrm{~mL}$ of alkaline aqueous phase. Data refer to complete substrate conversion.

Table 2. Hydrodehalogenation of 4-Chloropropiophenone 1 under Multiphase Conditions in the Presence of a Buffered Aqueous Phase ${ }^{a}$

\begin{tabular}{cccrccccc}
\hline & & \multicolumn{6}{c}{ products (\% by GC) } \\
\cline { 3 - 8 } run & buffer pH & time (h) & $\mathbf{2}$ & $\mathbf{4}$ & $\mathbf{5}$ & $\mathbf{7}$ & $\mathbf{8}$ & $\mathbf{7}+\mathbf{8}$ \\
\hline 1 & 7.00 & 7 & 9 & 46 & & 9 & 17 & 26 \\
2 & 5.00 & 7 & 14 & 35 & 9 & 18 & 27 \\
3 & 2.00 & 6 & 0 & 24 & 18 & 24 & 42 \\
4 & 1.00 & 6 & 0 & 8 & 16 & 33 & 49
\end{tabular}

a Conditions: $\mathrm{T}=50{ }^{\circ} \mathrm{C} ; \mathrm{Cl} / \mathrm{Pt}=32.6 ; \mathrm{Pt} / \mathrm{C}$ (5\%) $0.0586 \mathrm{~g} ; 7$ $\mathrm{mL}$ of $0.07 \mathrm{M} \mathbf{1}$ in isooctane; aqueous phase buffered at $\mathrm{pH} 7.00$, $5.00,2.00,1.00$. Data refer to complete substrate conversion.

$\mathbf{2}$ is reached, at complete substrate conversion. We report only the data referring to the amounts of PT agent found to afford the highest sel ectivity, which were determined experimentally by running a series of reactions. All data were verified by conducting each reaction at least three times. As was previously reported, by using $50 \% \mathrm{KOH}_{\mathrm{aq}},{ }^{5}$ the selectivity toward $\mathbf{4}$ was high (94\% for A336, entry 1 ; and $95 \%$ for TEBA, entry 2) and p-chlorophenylpropanol $\mathbf{3}$ was the byproduct (6\% for $\mathbf{A} 336$ and 5\% for TE BA). By lowering the $\mathrm{KOH}_{\text {aq }}$ concentration from $50 \%$ to $5 \%$, the hydrodehal ogenation reaction of $\mathbf{1}$ still afforded the dehalogenated aryl alcohol, although with lower selectivity compared to the preceding case: after $6 \mathrm{~h}$, using A336 and TEBA, the yield of $\mathbf{4}$ was $85 \%$ and $80 \%$, respectively (entries 3 and 4). Interestingly, the dehalogenated aryl ketone $\mathbf{2}$ was now the main other product (12\% and $15 \%$ with $\mathbf{A} 336$ and TEBA, respectively), and no p-chlorophenyl propanol $\mathbf{3}$ was observed.

Figure 1 shows the progress of the hydrodehalogenation reaction of $\mathbf{1}$ in the presence of $5 \% \mathrm{KOH}_{\mathrm{aq}}$, under the conditions described in entry 3 of Table 1.

The fact that ketone $\mathbf{2}$, and not p-chlorophenyl propanol 3, was observed upon lowering the $\mathrm{KOH}_{\mathrm{aq}}$ concentration from $50 \%$ to $5 \%$ confirmed our previously reported hypothesis ${ }^{5}$ of two distinct pathways for the reaction of 1 to 4 (Scheme 1).

In the case of $\mathrm{KOH}_{\text {aq }} 50 \%$ the rates of the two parallel reactions (path "a" and path " $b$ ") were competitive, and the key to reduce unwanted byproducts was to favor path "a" by adjusting the platinum/PT agent ratio and the reaction time. Instead, in the case of $5 \% \mathrm{KOH}_{\mathrm{aq}}$, path "a" was faster than path " $b$ " and the reaction afforded $\mathbf{2}$ without concomitant formation of 3; the ketone $\mathbf{2}$ then further reacted to give 4 selectively. These results indicated that the selectivity toward dehalogenation could be controlled by a combined effect of the Pt/PT agent ratio and of the concentration of $\mathrm{KOH}$. In other words, the presence of the base not only minimized the

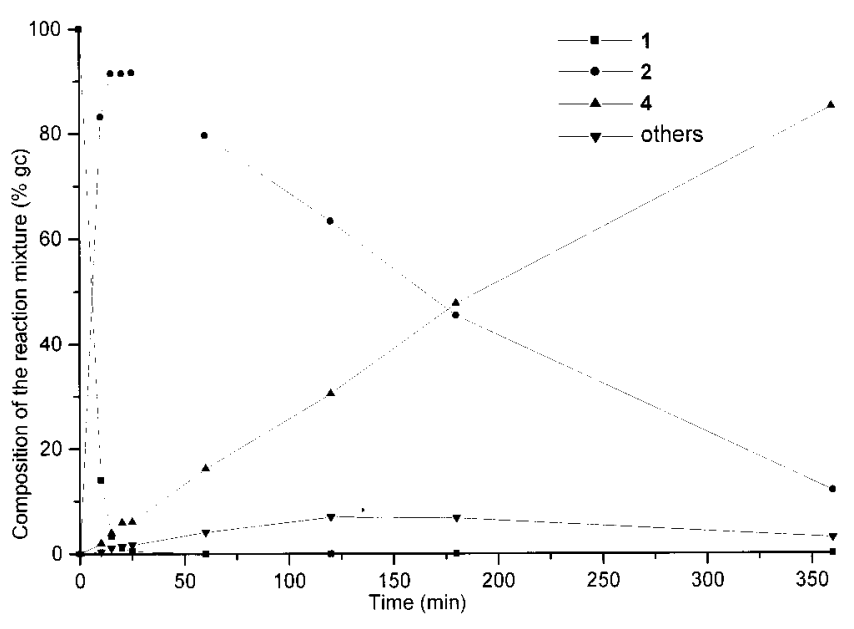

Figure 1. Multiphase hydrodechlorination of 4-chloropropiophenone in the presence of $5 \% \mathrm{KOH}$ (entry 3, Table 1).

\section{Scheme 1}

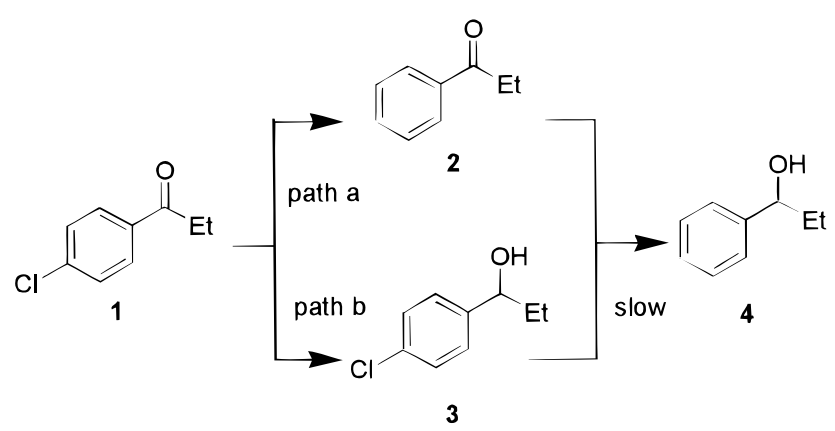

negative effects of the $\mathrm{HCl}$ formed during the hydrodehalogenation reaction ${ }^{6-8}$ but also allowed for control of the reaction selectivity simply by accelerating the rate of path "a" (hydrodehalogenation of the aryl ring) with respect to " $b$ " (carbonyl hydrogenation), and not by modifying the reaction pathway.

A further and more convincing evidence for this behavior was gathered by conducting the hydrodehalogenation of $\mathbf{1}$ with $1 \% \mathrm{KOH}_{\mathrm{aq}}$. Under such conditions, the reaction could be selectively and easily directed toward the dehalogenated aryl ketone $\mathbf{2}$. At complete substrate conversion (after $17 \mathrm{~min}$ ) we observed $95 \%$ and $89 \%$ of 2, using A336 (entry 5) and TEBA (entry 6), respectively, the remaining product being 4 (3\% and $10 \%$, respectively). In this case, by merely diminishing the concentration of the base, chlorine removal took place without any further reduction process. This fact indicated that the rate of conversion of $\mathbf{1}$ into $\mathbf{2}$ was fast relative to the successive carbonyl hydrogenation to afford 4 . Figure 2 shows the progress of the reaction with $1 \% \mathrm{KOH}_{\mathrm{aq}}$ described in entry 5 of Table 1 . By carefully monitoring the reaction, the formation of $\mathbf{4}$ could be prevented, and with $1 \% \mathrm{KOH}_{\text {aq }}$ the unwanted byproducts were kept below $3 \%$.

As we previously noted, ${ }^{5}$ it is a synergistic effect between the presence of the base and of the onium salt which accounts for the chemoselective outcome of the multiphase hydrodechlorination. In fact, when the multi-

(6) Hoke, J . B.; Gramiccioni, G. A.; Balko, E. N. Appl. Catal. B: Env. 1992, 1, 285

(7) Pasett, P.; Gentile, F. J . Org. Chem. 1995, 60, 1351.

(8) Armandia, M. A.; Borau, V.; J imenez, C.; Marinas, J. M. Sempere, M. E.; Urbano, P. Appl. Catal. 1988, 43, 47. 


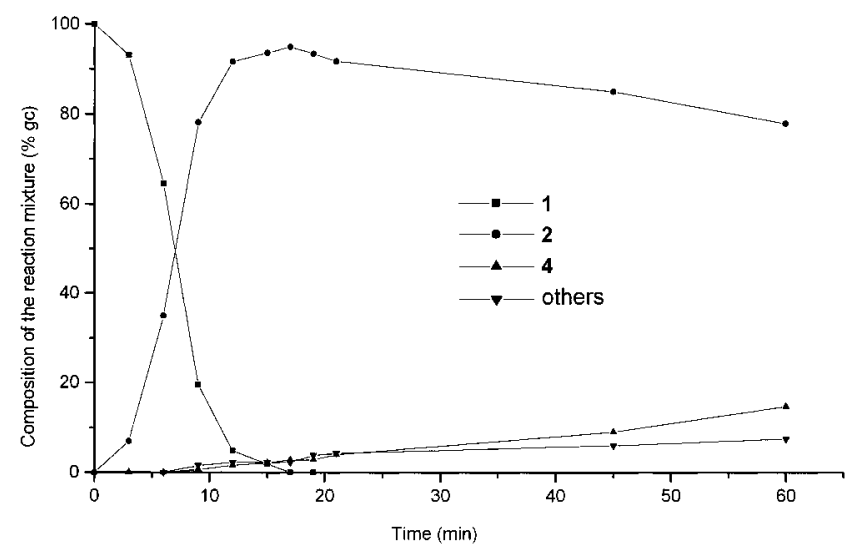

Figure 2. Multiphase hydrodechlorination of 4-chloropropiophenone in the presence of $1 \% \mathrm{KOH}$ (entry 5, Table 1).

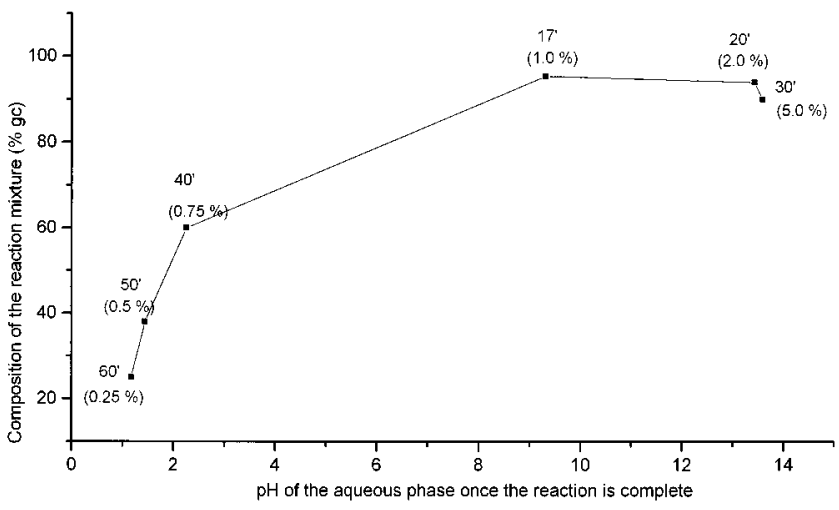

Figure 3. Multiphase hydrodechlorination of 4-chloropropiophenone: selectivity toward $\mathbf{2}$ at different $\mathrm{KOH}$ concentrations and at complete substrate conversion.

phase hydrodehal ogenation of 4-chloropropiophenone was carried out in the presence of $1 \% \mathrm{KOH}$, but without the PT agent, the chemoselectivity of the reaction was sharply lowered: after 17 min conversion was complete, but we observed only $51 \%$ of $\mathbf{2}$, along with $31 \%$ of $\mathbf{4}, 11 \%$ of propylbenzene $\mathbf{6}$, and $7 \%$ of aliphatic products (entry 7, Table 1). On the other hand, we al ready reported that in the absence of the base, only a range of aliphatic products was observed, both with and without a PT agent. ${ }^{5}$

2. pH Effect. These facts allowed us to conclude that the decrease of the $\mathrm{KOH}_{\text {aq }}$ concentration from $50 \%$ to $1 \%$ had a dramatic effect on the reaction selectivity: in particular, at $\mathrm{KOH}_{\mathrm{aq}}=1 \%$ and by tuning the amount of the PT agent, the halo ketone 1 could be hydrodechlorinated with up to $95 \%$ selectivity without concurrent reduction of the carbonyl function.

To investigate more indepth the effect of the base concentration on selectivity, a series of reactions was then conducted by further progressively lowering the $\mathrm{KOH}_{\mathrm{aq}}$ concentration from $1 \%$ to $0.25 \%$ and by measuring the $\mathrm{pH}$ of the aqueous phase once the substrate was completely converted. Then the hydrodehalogenation of $\mathbf{1}$ was also conducted in the absence of $\mathrm{KOH}$ in the aqueous phase, and an acidic final pH of 1.0 was measured, consistent with $\mathrm{HCl}$ produced by the reaction being quantitatively absorbed in the aqueous phase. The results are shown in Figure 3, where the maximum selectivity toward $\mathbf{2}$ (\% yield by GC, after the indicated time) is plotted against the final $\mathrm{pH}$ of the aqueous phase.
The final pH was chosen as the reference parameter since it provides a measure for the effect on selectivity of the $\mathrm{KOH}_{\text {aq }}$ concentration. All data refer to complete substrate conversion. To allow a faster interpretation of the results, the different base concentrations are shown in parentheses. The observed trend reaches a maximum yield of $2(95 \%)$ in correspondence with a $\mathrm{KOH}_{\text {aq }}$ concentration of $1 \%$. As can be seen, if the $\mathrm{KOH}_{\text {aq }}$ concentration was $2 \%$ or higher, the final $\mathrm{pH}$ of the reaction remained $>$ 13.5, implying that the $\mathrm{HCl}$ developed by the reaction was neutralized by the base, which remained in excess. By using $1 \% \mathrm{KOH}_{\mathrm{aq}}$ the final $\mathrm{pH}$ of the aqueous phase was still alkaline; however during the reaction it decreased from 13.2 to $9.4 \mathrm{pH}$ units, at complete conversion after $17 \mathrm{~min}$, and the selectivity toward 2 reached 95\%. From this value onward of $\mathrm{KOH}_{\mathrm{aq}}$ concentration, a further decrease of the base amount caused the final $\mathrm{pH}$ of the aqueous phase to turn acidic. Thus, using $\mathrm{KOH}_{\mathrm{aq}}$ $0.75 \%, 0.50 \%$, and $0.25 \%$, a pH of $2.26,1.44$, and 1.17 , respectively, was measured after complete dechlorination, and the selectivity toward 2 dropped to $60 \%, 38 \%$, and $25 \%$, respectively.

Hence, the rate of formation of the products was strictly dependent on the alkalinity of the aqueous phase: this allowed us to tune the chemoselectivity of the multiphase catalytic hydrodehal ogenation reaction. At low base concentrations $\left(1 \% \leq \mathrm{KOH}_{\mathrm{aq}} \leq 2 \%\right)$, the rate of formation of the dehalogenated ketone $\mathbf{2}$ was faster than the successive carbonyl reduction, while higher concentrations of $\mathrm{KOH}$ promoted the rapid formation of the dehalogenated alcohol 4. If one refers to path "a" of Scheme 1, when $\mathrm{KOH}_{\text {aq }}$ was used in the range of 1 to $2 \%$, the first step $\mathbf{1} \rightarrow \mathbf{2}$ was faster than the second one $(\mathbf{2} \rightarrow \mathbf{4})$; while when using a higher $\mathrm{KOH}_{\mathrm{aq}}$ concentration (50\% to $5 \%$ ) the second step $\mathbf{2} \rightarrow \mathbf{4}$ competed with the first step $\mathbf{1} \rightarrow \mathbf{2}$. Therefore, an increase of the $\mathrm{KOH}_{\mathrm{aq}}$ concentration caused a major enhancement of the rate of carbonyl hydrogenation over the rate of aryl dehalogenation.

To obtain a good control of chemoselectivity, the reaction environment had to remain alkaline until after complete substrate conversion. This fact indicated that the lower limit of the amount of $\mathrm{KOH}_{\mathrm{aq}}$ necessary to achieve good selectivities was approximately the minimum concentration required to ensure the complete neutralization of the $\mathrm{HCl}$ formed in the hydrodehal ogenation reaction, in our case $1 \%$. It was also in agreement with the observation, reported by others, ${ }^{8}$ that the presence of a base inhibits carbonyl hydrogenolysis of acetophenone, in favor of its reduction to the corresponding alcohol.

Further evidence for the alkalinity-dependent chemoselectivity trend in the hydrodehal ogenation of $\mathbf{1}$ derived from our previous observation that, when using a neutral aqueous phase in the presence of $\mathbf{A} 33 \mathbf{6}$ (which caused the final $\mathrm{pH}$ of the reaction mixture to become acidic: $\mathrm{pH}$ $=1.0$ ), the sel ectivity toward $\mathbf{2}$ and $\mathbf{4}$ decreased, interestingly in favor of products arising from the reduction of the aromatic ring: thus, cyclohexyl ethyl ketone 7 (21\%) and cycl ohexylpropanol 8 (44\%) were obtained, which did not react further. ${ }^{5}$ This preferential reduction of the aryl ring seemed interesting al so from a synthetic standpoint. It was only observed when $\mathbf{A} \mathbf{3 3 6}$ was used in the absence of $\mathrm{KOH}_{\mathrm{aq}}$; instead, mainly propylcyclohexane $\mathbf{5}$ was obtained by removing the PT agent. It should be noted that products $\mathbf{7}$ and $\mathbf{8}$ appeared rapidly during the 
Table 3. Hydrodehalogenation of 4-Chloropropiophenone 1 under Multiphase Conditions in the Presence of Different Aminesa

\begin{tabular}{cccccc}
\hline & & & \multicolumn{3}{c}{ products (\% by GC) } \\
\cline { 3 - 6 } run & \multicolumn{1}{c}{ amine } & time $(\min )$ & $\mathbf{2}$ & $\mathbf{4}$ & others \\
\hline 1 & $\mathrm{Et}_{2} \mathrm{NH}$ & 55 & 83 & 15 & 2 \\
2 & $\mathrm{Et}_{3} \mathrm{~N}$ & 45 & 83 & 15 & 2 \\
3 & $\mathrm{n}-\mathrm{Bu}_{3} \mathrm{~N}$ & 105 & 82 & 16 & 2 \\
4 & $\left(\mathrm{PhCH}_{2}\right)_{3} \mathrm{~N}$ & 60 & 83 & 16 & 1 \\
5 & $\mathrm{n}-\mathrm{C}_{8} \mathrm{H}_{17} \mathrm{NH}_{2}$ & 55 & 82 & 15 & 3
\end{tabular}

a Conditions: $\mathrm{T}=50^{\circ} \mathrm{C}$; Pt/C $5 \% 0.010 \mathrm{~g}$; amine used in $0.2 \mathrm{M}$ ration with respect to $1 ; 0.07 \mathrm{M}$ solution of $\mathbf{1}$ in isooctane $(7 \mathrm{~mL})$; aqueous phase $\mathrm{KOH} 1 \%(4 \mathrm{~mL})$. Data refer to complete substrate conversion.

reaction, indicating that the observed chemoselectivity depended specifically on the alkalinity of the aqueous phase and was therefore not determined simply by different reaction rates.

This fact, and the effect of the $\mathrm{pH}$ on selectivity discussed earlier, prompted us to investigate the influence of acidic $\mathrm{pH}$ 's on the outcome of the hydrodehalogenation reaction of $\mathbf{1}$ in the presence of $\mathbf{A 3 3 6}$. Under phase-transfer catalysis conditions, the catalytic reduction of aromatic ketones to yield alicyclic ketones and alcohols was claimed in the presence of $[1,5-\mathrm{HDRhCl}]_{2}$ as a homogeneous catalyst and of a buffered aqueous solution. ${ }^{9}$ Therefore, a series of reactions was conducted, with an aqueous phase buffered at $\mathrm{pH}=1.00,2.00,5.00$, and 7.00; the experiments were run under the conditions similar to those used for the reaction performed with pure water (unbuffered solution) where a combined yield of $65 \%$ was reached for $\mathbf{7}+\mathbf{8} .{ }^{5}$ The results are collected in Table 2.

As expected, at the $\mathrm{pH}$ 's tested, the ketone reduction became competitive with aromatic ring reduction. Even though the trend confirmed that lower final pH's $(\mathrm{pH}<$ 1) favored ring reduction products (compare entries 1-4 of Table 2), a selectivity as high as that with pure water (65\% combined), toward cyclohexyl ketone 7 and cyclohexyl alcohol 8, was never reached. In no case was $\mathbf{5}$ observed. It was therefore concluded that, when using pure water, the gradual decrease of $\mathrm{pH}$ during the progress of the reaction promoted reduction of the aryl moiety, while protecting the carbonyl function. Therefore, in this case also, the effect of $\mathrm{pH}$ on chemoselectivity appears to be important.

3. E ffect of the PT Agent. While onium salts, even of aliphatic amines, are preferentially soluble in the aqueous phase, a favorable partitioning of aliphatic amines into the organic phase should, on the other hand, be expected under multiphase conditions. Therefore, if an amine was used, in place of an onium salt, a modification of the catalyst surface present in the organic phase should be expected as well, and a consequent effect on the chemoselectivity of the reaction should be observable. To this aim, amines with different solubility properties in the aqueous and organic phases were chosen: diethyl-, triethyl-, tributyl-, tribenzyl-, and octylamine were tested in a $0.2 \mathrm{M}$ ratio with respect to 4-chloropropiophenone 1. Table 3 reports the results. Since under the usual conditions conversion of $\mathbf{1}$ was complete after $17 \mathrm{~min}$, in order to follow with higher accuracy any variations of the reaction outcome, a lower mol \% of Pt catalyst (5 times

(9) J anuszklewicz, K. R.; Alper, H. Organometallics 1983, 2, 1055.

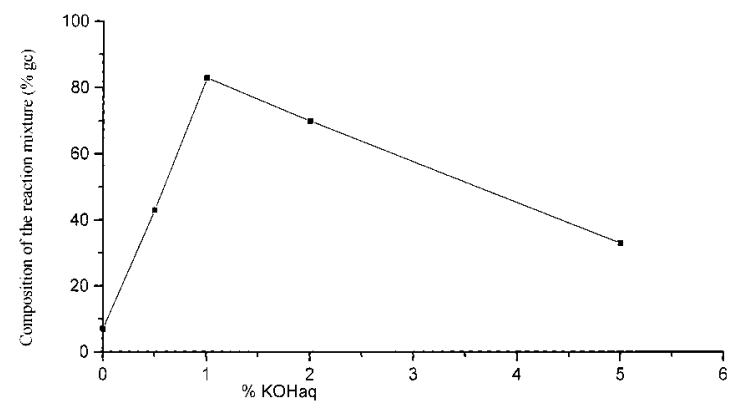

Figure 4. Multiphase hydrodechlorination of 4-chloropropiophenone: selectivity toward $\mathbf{2}$ at different $\mathrm{KOH}$ concentrations using triethylamine as modifier of the catalyst surface.

lower than usual) was used. In this way, the reaction was slowed and complete consumption of the substrate required 45-105 min (entries 1-5); in the presence of 1\% $\mathrm{KOH}_{\mathrm{aq}}$, the use of different amines allowed the dehalogenation to occur with selectivities of $80-83 \%$ toward the ketone 3. Although the reaction was not as chemoselective as in the case of the onium salts, it followed a trend similar to the one observed using $\mathbf{A} \mathbf{3 3 6}$. This behavior was further confirmed by running the hydrodechlorination reaction in the presence of triethylamine, with varying $\mathrm{KOH}_{\mathrm{aq}}$ concentrations. The results are plotted in Figure 4. Similar to what was observed previously (compare Figure 3 ), the reaction outcome was markedly dependent upon the $\mathrm{pH}$ of the aqueous phase: in particular, the highest selectivity toward the formation of $\mathbf{3}$ was obtained with $1 \% \mathrm{KOH}_{\text {aq }}$. Instead, when the concentration was either reduced or increased the selectivity dropped, and ketone hydrogenation took over. While, the lipophilicity of the amine appeared to have no appreciable effect on directing the chemoselectivity of the reaction: both hydrophilic diethylamine and hydrophobic tribenzylamine afforded almost identical yields of $\mathbf{3}$ and $\mathbf{4}$ after 55 min of reaction $(82-83 \%$ and $15 \%$ : Table 3 , entries 1 and 5, respectively). Therefore, it seems that the concentration and the interaction of the PT agent with the supported metal catalyst, rather than its partitioning (or lipophilicity) between the two phases, are the key factors which determined the observed reaction pathway.

4. Mechanism of Action of the PT Agent. As was previously mentioned, before addition of the PT agent to the aqueous-organic system, the carbon-supported metal catalyst was uniformly distributed as a suspension in both phases. Instead, once the PT agent was added, the Pt (or Pd)/C catalyst appeared to become coated by it, and to settle at the phase boundary at rest; upon stirring it was completely transferred into the organic phase.

The interactions between the carboxyl and carbonyl groups present on the active carbon surface ${ }^{10}$ and the PT agent (onium salt) formed a lipophilic film on the Pt/C catalyst, thereby favoring its partitioning into the organic phase where the reaction takes place, and probably generating a suitable microenvironment for the reaction.

Under basic conditions, the first step in Scheme 2 takes place in the aqueous phase and gives the formation of carboxylate anions on the surface of the carbon support of the Pt catalyst. In the second step, exchange of $\mathrm{K}^{+}$with the cation $\left(\mathrm{Q}^{+}\right)$of the onium salt (or with the ammonium nitrogen, $\mathrm{HNR}_{3}{ }^{+}$, in the case of the amine) makes the catalyst surfacelipophilic. The supported catalyst is then

(10) Swiatkowskj, A.; Wieczorek, M. Chem. Anal. 1984, 25, 565. 


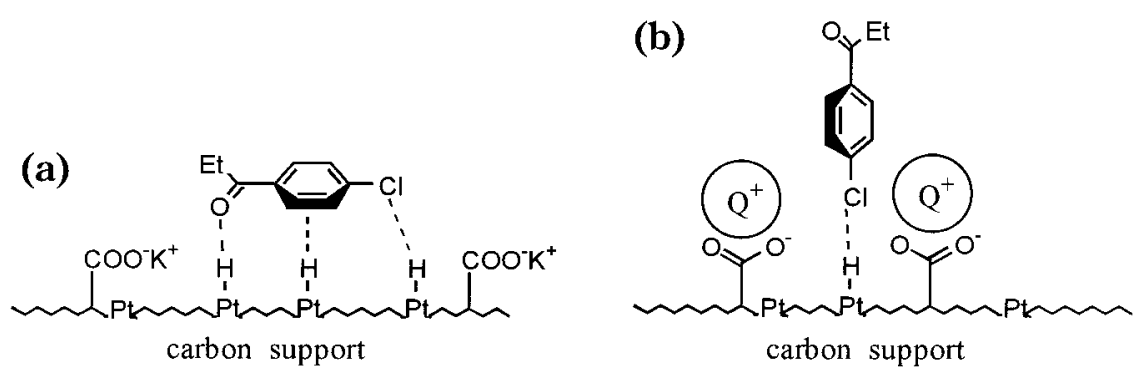

Figure 5. Pictorial representation of the hypothetical arrangement of substrate $\mathbf{1}$ on the catalyst surface in the absence of (a) and in the presence of (b) the onium salt $\mathrm{Q}^{+}$.

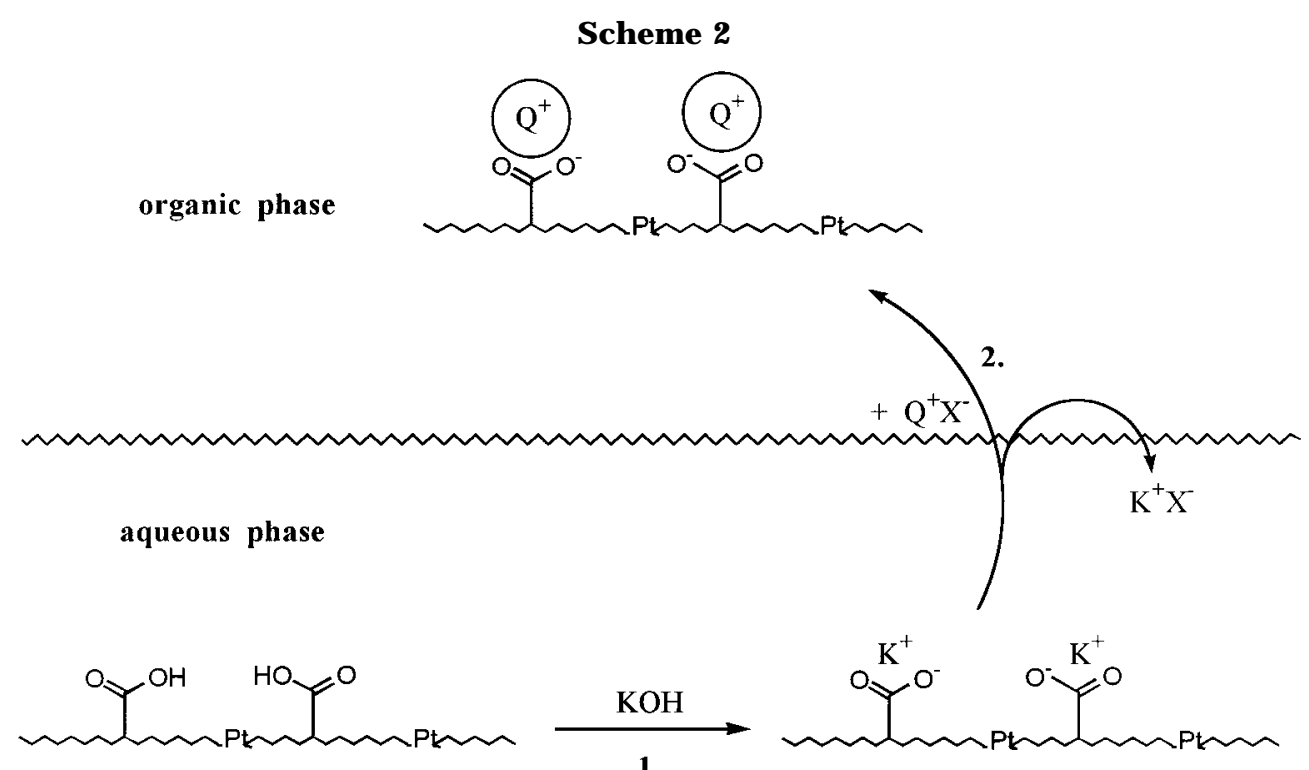

"extracted"11 into the organic phase, thereby favoring formation of the $-\mathrm{COO}^{-} \mathrm{Q}^{+}$species (Scheme 2). The same shift does not occur under neutral or acidic conditions.

The large lipophilic onium cation $\mathrm{Q}^{+}$modifies the catalyst because, by completely coating the carbon surface, it finds itself near the active Pt sites.

This change might directly affect the selectivity of the hydrodehalogenation reaction of the substrate in two ways: (a) by an interaction of $\mathrm{Q}^{+}$with the incoming substrate, i.e. by modifying its "solvation shell" near the catalyst and favoring approach and reduction of the haloaromatic portion of the substrate (Figure $5 a, b)$; (b) by an effect on the coordination sphere of Pt due to the proximity of $\mathrm{Q}^{+}$which changes its catalytic activity.

Therefore, the modified catalyst surface may interact with the substrate in a way that provides preferential reduction of one functional group respect to another (e.g. hydrodehalogenation vs CO reduction).

\section{Conclusions}

Under the here reported multiphase conditions, the outcome of the Pt-catalyzed hydrodehalogenation of 4-chloropropiophenone 1 could be di rected to afford either 1-phenyl-1-propanol $\mathbf{4}$ or phenyl ethyl ketone $\mathbf{2}$ selectively, simply by appropriately tuning the alkalinity of

(11) In analogy to the proposed mechanism for extraction of carboxylic acids into an organic phase by a PT catalyst in the presence of $\mathrm{OH}^{-}$. Cf. Dehmlow, E. V.; Dehmlow, S. S. Phase Transfer Catalysis. In Monographs in Modern Chemistry, 2nd rev. ed.; Ebel, H. F., Ed.; Weinheim; Deerfield Beach, FL; Basel: Verlag Chemie, 1983; pp 3241 and refs 34 and 35 therein. the aqueous phase, provided that a suitable onium salt or aliphatic amine was present. An acidic aqueous phase directed chemoselectivity toward the formation of products arising from aromatic ring reduction $\mathbf{7}$ and $\mathbf{8}$ rather than ketone hydrogenolysis, but a buffered acidic phase did not improve the previously reported yields.

In addition to preventing catalyst poisoning by transferring the $\mathrm{HCl}$ to the alkaline aqueous phase where it is neutral ized, the PT agent appeared to coat the partides of supported metal catalyst, thereby transferring it to the organic phase where the reaction took place. The polar PT agent film formed on the Pt/C catalyst, thus providing a reaction microenvironment for the substrate, with functional group-specific catalytic sites.

The multiphase system therefore offers a wide versatility since it allows to tune sel ectivity by changing simple parameters such as $\mathrm{KOH}_{\text {aq }}$ concentration, PT agent, and reaction conditions. It thus offers the opportunity for new synthetic applications, thanks also to the facile separation of the products and to the small amounts of reagents required.

In this regard, it should be pointed out that the selectivity effect, which is obtainable by adding the PT agent, is very large relative to the amount which is used, making its efficiency very high. By forcing the reaction to take place in this microenvironment, made up by the supported metal catalyst and the PT agent, one is effectively steering it precisely to the reactive site.

F urther investigations are underway to take advantage of these features for synthetic applications, e.g. to employ the hydrodehal ogenation of hal oar omatic ketones for the 
formation of products arising from aromatic ring reduction (compare $\mathbf{7}$ and $\mathbf{8}$ ) of substituted haloaromatics.

\section{Experimental Section}

All reagents and solvents were ACS grade and were used without further purification. The $5 \% \mathrm{Pt} / \mathrm{C}$ was from Fluka, Art. no. 80982; its surface area was $700-800 \mathrm{~m}^{2} / \mathrm{g}$. GC analyses were performed on a Varian GC 3400 using a fused silica capillary column ( $30 \mathrm{~m} \times 0.25 \mathrm{~mm}$ ) with SPB-5 as the liquid phase (film thickness $0.25 \mathrm{~mm}$ ). GC/MS analyses were performed on a HP 5971 mass detector coupled to a HP 5890 gas chromatograph fitted with a $30 \mathrm{~m} \times 0.25$ DB5 capillary column.

The structure of all compounds obtained (2-8) was confirmed by mass spectroscopy.

General Procedure for the Hydrodehalogenation Reactions. All reactions were carried out in a $25 \mathrm{~mL}$ threenecked flask equipped with a condenser and a system for the bubbling of hydrogen.

If not otherwise indicated, they were carried out on $7.0 \mathrm{~mL}$ of an isooctane solution of the 4-chloropropiophenone $\mathbf{1}$ (0.49 $\mathrm{mmol}$ ), $4.0 \mathrm{~mL}$ of aqueous solution (alkaline by $\mathrm{KOH}$ or at controlled $\mathrm{pH}$ by using a buffer solution $\left.{ }^{12}\right), 0.0586 \mathrm{~g}$ of $5 \% \mathrm{Pt} / \mathrm{C}$ $(0.015 \mathrm{mmol}$ of $\mathrm{Pt})$, and $0.0725 \mathrm{~g}$ of PT agent $(0.20 \mathrm{mmol})$.

Hydrogen was bubbled at atmospheric pressure through the organic phase at a rate of about $1 \mathrm{~mL} / \mathrm{min}$. The reaction flask was thermostated at $50 \pm 0.1{ }^{\circ} \mathrm{C}$, and the mixture was magnetically stirred at $1000 \mathrm{rpm}$ with a bar $2.5 \mathrm{~cm}$ in length and $0.6 \mathrm{~cm}$ in diameter.

The reaction course was followed by gas chromatography. Conversions are referenced to the internal standard (n-decane).

Acknowledgment. This work was supported by INCA (Interuniversity Consortium Chemistry for the Environment) and MURST (Ministero dell'Università e Ricerca Scientifica e Tecnologica), F ondo 40\%.

\section{J O982337D}

(12) Buffer solutions were prepared as follows: $\mathrm{pH}=1.00: 0.13 \mathrm{M}$ $\mathrm{HCl} / 0.050 \mathrm{M} \mathrm{KCl} ; \mathrm{pH}=2.00: 0.03 \mathrm{M}$ citric acid/0.0082 $\mathrm{M} \mathrm{HCl} / 0.061$ $\mathrm{M} \mathrm{NaCl} ; \mathrm{pH}=5.00$ : $0.096 \mathrm{M}$ citric acid/0.20 M NaOH.; $\mathrm{pH}=7.00$ : $0.029 \mathrm{M} \mathrm{NaOH} / 0.050 \mathrm{M} \mathrm{KH}_{2} \mathrm{PO}_{4}$. 